\title{
A NOTE ON COMPACT SEMIRINGS
}

\author{
JOHN SELDEN
}

By a topological semiring we mean a Hausdorff space $S$ together with two continuous associative operations on $S$ such that one (called multiplication) distributes across the other (called addition). That is, we insist that $x(y+z)=x y+x z$ and $(x+y) z=x z+y z$ for all $x, y$, and $z$ in $S$. Note that, in contrast to the purely algebraic situation, we do not postulate the existence of an additive identity which is a multiplicative zero.

In this note we point out a rather weak multiplicative condition under which each additive subgroup of a compact semiring is totally disconnected. We also give several corollaries and examples.

Following the notation current in topological semigroups we let $H[+](e)$ represent the maximal additive subgroup containing an additive idempotent $e$. Similarly $H[\cdot](f)$ will denote the maximal multiplicative group of a multiplicative idempotent $f$. The minimal closed additive or multiplicative semigroup containing $x$ is denoted by $\Gamma[+](x)$ or $\Gamma[\cdot](x)$ respectively. By $E[+]$ or $E[\cdot]$ we mean the collection of additive or multiplicative idempotents. Finally $A^{*}$ represents the topological closure of $A$. For references on the properties of these sets the reader may see [1].

TheOREM. If $S$ is a compact semiring such that $E[\cdot] S \cup S E[\cdot]=S$ then each additive subgroup of $S$ is totally disconnected.

Proof. Let $S$ be a compact semiring such that $E[\cdot] S \cup S E[\cdot]=S$ and let $G$ be an additive subgroup with identity $e$. Suppose $G$ is not totally disconnected. Then neither is $H[+](e)$ since $G \subset H[+](e)$. That is, $C$, the component of $e$ in $H[+](e)$, is nontrivial. Now $C$ is a compact connected nontrivial group. It is well known [2, pp. 175, 190 , and 192] that $C$ must contain a nontrivial additive one parameter group $T$. Pick $t$ different from $e$ in $T$. Recall that $t \in f S$ or $S f$ for some $f$ in $E[\cdot]$. Suppose $t \in f S$. Clearly $f S$ is a compact subsemiring for which $f$ is a multiplicative left identity. Thus $f t=t$ so $f T$ is nontrivial and of course, $f T$ is connected. Therefore $f S$ contains a connected nontrivial group. Similarly if $t \in S f$ then $S f$ is a compact subsemiring with right identity containing a connected nontrivial subgroup. Thus, without loss of generality, we may assume $S$ has a left or a right identity 1 .

Suppose 1 is a left identity. We identify each positive integer $n$ with

Received by the editors April 1, 1963. 
the $n$-fold sum of 1 . Thus, if $x \in S$, we may regard $n x$ as a product as well as a sum in $S$. Now, for each positive integer $n$, we have $n T=T$ so $(n T)^{*}=T^{*}$. This and the compactness of $S$ gives us $n T^{*}=T^{*}$. From a theorem of A. D. Wallace [3], we see that $x T^{*}=T^{*}$ for each $x$ in $\Gamma[+](1)$. But, $\Gamma[+](1)$ contains an additive idempotent g. Thus $g T^{*}=T^{*}$. On the other hand the additive idempotents $E[+]$ form a multiplicative ideal, so $T^{*} \subset E[+]$. Thus the additive group $T$ must consist of a single element. This is a contradiction. Since a similar argument applies in case $S$ contains a right identity, the theorem is proved.

By a clan we mean a compact connected semigroup with identity. Furthermore, we say a space $S$ is acyclic provided $H^{n}(S)=0$ for each positive integer $n$ where $H^{n}(S)$ represents the $n$th Cech cohomology group of $S$.

CoRollary 1. If $S$ is a compact semiring such that $E[\cdot] S \cup S E[\cdot]$ $=S$ then every additive subclan is acyclic.

Proof. Let $T$ be an additive subclan of $S$. Let $e$ be an additive idempotent of the minimal additive ideal of $T$. Then it is known that $e+T+e$ is a group and $H^{n}(T)=H^{n}(e+T+e)$ for each positive integer $n$ [4]. Now, by the theorem, $e+T+e$ is totally disconnected. But $e+T+e$, being a continuous image of $T$, is connected. Thus $e+T+e$ is a single point and $H^{n}(T)=0$ for $n>0$.

A semigroup $S$ is said to be normal if $S x=x S$ for each $x$ in $S$.

Corollary 2. Let $S$ be a compact semiring such thiat $S E[\cdot] \cup E[\cdot] S$ $=S$. Let $T$ be an additive subsemigroup of $S$.

(i) If $T$ is a continuum then the minimal ideal of $T$ is idempotent.

(ii) If also $T$ is normal and $(E[+] \cap T)+T=T$ then each closed ideal of $T$ is acyclic.

(iii) If also $T$ is metric then $T$ is arcwise connected.

(iv) If also $T=S$ then there is an element $k$ in $S$ such that $k+S$ $=S+k=k=k^{2}$.

Proof. Let $S$ be a compact semiring such that $S E[\cdot] \cup E[\cdot] S=S$ and $T$ be an additive semigroup of $S$. Suppose $T$ is a continuum. It is well known [5] that the minimal ideal of $T$ is the union of groups each of which is of the form $e+T+e$ where $e \in E[+] \cap T$. Now $e+T+e$, being a continuous image of $T$, is a continuum. But each additive subgroup of $S$ must be totally disconnected. Consequently, $e+T+e$ is a single point and we have shown that the minimal ideal of $T$ consists entirely of idempotents.

Suppose also that $T$ is normal and $(E[+] \cap T)+T=T$. The nor- 
mality of $T$ implies that its minimal ideal is a group. Since the minimal ideal of $T$ is also idempotent it must be a single point, say $k$. Now Corollary 3 of [6] gives us that each closed ideal of $T$ is acyclic and part (ii) is proved.

Assume in addition that $T$ is metric and select an additive idempotent $f$ of $T$. Clearly $f+T$ is a compact connected additive subsemigroup of $T$. Furthermore $k \in f+T$ and, because $f+T=T+f, f$ is an additive identity for $f+T$. Pick $x$ in $f+T$. We have $x+(f+T)=(x+f)$ $+T=x+T=T+x=T+(f+x)=(T+f)+x=(f+T)+x$. Thus $f+T$ is additively normal. Also, by the theorem, each additive subgroup of $f+T$ is totally disconnected. Now R. P. Hunter has shown that each such semigroup contains an arc (indeed, an $I$-semigroup) from the zero to the identity [7, Theorem 1]. Since $f+T$ is metric it is arcwise connected. But $T=U\{f+T \mid f \in E[+] \cap T\}$ and $k \in f+T$ for each $f$ in $E[+] \cap T$. Therefore $T$ is arcwise connected.

Suppose $T=S$ so that $S+k=k+S=k$. Now $k$ is in either $E[\cdot] S$ or $S E[\cdot]$. In the former case there is a $g$ in $E[\cdot]$ such that $g k=k$. But because $k$ is an additive zero we have $k=g+k$ and $k=k+k^{2}$. Thus $k=k+k^{2}=(g+k) k=k^{2}$. In case $k \in S E[\cdot]$ a similar argument gives us that $k=k^{2}$ and the corollary is proved.

Recall that if the minimal ideal $K$ of a compact semigroup $S$ is idempotent then its structure is completely known [8]. That is, $K$ must be of the form $A \times B$ where multiplication is defined by $(a, b) \cdot\left(a^{\prime}, b^{\prime}\right)=\left(a, b^{\prime}\right)$ for any $a$ and $a^{\prime}$ in $A$ and $b$ and $b^{\prime}$ in $B$.

If $S$ is a semiring, let $H[+]$ denote the union of all the additive subgroups of $S$.

CoROllary 3. Let $S$ be an additively commutative semiring such that $S E[\cdot] \cup E[\cdot] S=S$. If $S$ is a metric continuum and $e \in E[+]$ then $E[+], E[+]+S, e+S, H[+]$ and $e+H[+]$ are arcwise connected.

Proof. Clearly $E[+]$ is a closed additive subsemigroup and a multiplicative ideal. Now, since $S$ is connected and $S E[\cdot] \cup E[\cdot] S$ $=S$, the multiplicative ideals of $S$ are connected and $E[+]$ is a continuum. From this it follows that $E[+]+S$ is also a continuum. Furthermore, $e+S$ being a continuous image of $S$, is a continuum. Applying the third part of Corollary 2, we have that $E[+], E[+]+S$, and $e+S$ are arcwise connected. Now suppose $x \in S$ and $G$ is an additive subgroup of $S$. It follows from the distributive property that $x G$ and $G x$ are additive subgroups of $S$. Thus $x H[+] \cup H[+] x \subset H[+]$. That is, $H[+]$ is a multiplicative ideal of $S$. It is well known [1] that $H[+]$ is a closed additive subsemigroup of $S$. Thus Corollary 2 gives us that $H[+]$ is arcwise connected. 
Notice that for $E[+]$ to be a continuum it is only necessary that $S$ be a continuum and $E[\cdot] S \cup S E[\cdot]=S$. In case $E[+]$ is a single point $k$ we have $k+S=S+k=k S=S k=k$. To see this, recall that $E[+]$ is a multiplicative ideal and hence must contain the minimal such. On the other hand, the minimal additive ideal of $S$ consists of idempotents and therefore must be $k$.

A semigroup is said to be simple if it contains no proper ideals.

CoROLlaRY 4. If $S$ is a compact connected additively simple semiring then each multiplicative idempotent of $S$ is an additive idempotent of $S$.

Proof. Let $e$ be a multiplicative idempotent of $S$. Then $e S$ is a compact connected subsemiring for which $e$ is a multiplicative left identity. Now $e S$ is additively simple since it is additively a homomorphic image of $S$. Thus the first part of Corollary 2 gives us that $e S$ is additively idempotent. But $e \in e S$ so $e$ is an additive idempotent and the corollary is proved.

ExAmple. Let $A$ be the field of integers mod 3 with the discrete topology and $B$ be the interval $[0,1]$. For $x$ and $y$ in $B$, define $x+y$ $=x y=\min \{x, y\}$. Note that $B$ is a semiring so that $A \times B$ becomes a semiring under coordinate-wise addition and multiplication. Define the equivalence relation $\alpha$ on $A \times B$ by: $(a, j) \alpha\left(a^{\prime}, j^{\prime}\right)$ if (1) $a=a^{\prime}$ and $j=j^{\prime}$ or (2) $j=j^{\prime}=0$. Clearly $\alpha$ is a closed congruence. Thus $(A \times B) / \alpha$ is a compact connected semiring with multiplicative identity. The maximal additive subgroups of $(A \times B) / \alpha$ are of the form $(A \times\{b\}) / \alpha$ and of course totally disconnected.

On the other hand let $C$ be the circle group written additively and given the multiplication $x y=0$ for all $x$ and $y$ in $C$. According to the theorem, $C$ can not be imbedded in a compact semiring with multiplicative identity (even if the identity is isolated).

QUESTION. Regarding the proof of the third part of Corollary 2, it is easily seen that $e+T$ is not only arcwise connected but also contractable. Indeed $(e+T) \cup(f+T)$ is contractable for $e$ and $f$ in $E[+] \cap T$. The referee has pointed this out and raised the question: Is $T$ contractable?

\section{REFERENCES}

1. A. D. Wallace, The structure of topological semigroups, Bull. Amer. Math. Soc. 61 (1955), 95-112.

2. D. Montgomery and L. Zippin, Topological transformation groups, Tracts in Mathematics 1, Interscience, New York, 1955. $1-3$.

3. A. D. Wallace, Inverses in Euclidean mobs, Math. J. Okayama Univ. 3 (1953), 43-54.

4. - Cohomology, dimension, and mobs, Summa Brasil. Math. 3 (1953), 
5. - A note on mobs. II, An. Acad. Brasil. Ci. 25 (1953), 335-336.

6. H. Cohen and R. J. Koch, Analytic semigroups and multiplications on 2-manifolds, Trans. Amer. Math. Soc. (to appear).

7. R. P. Hunter, Certain upper semi-continuous decompositions of a semigroup, Duke Math. J. 27 (1960), 283-289.

8. A. D. Wallace, The Rees-Suschkewitsch structure theorem for compact simple semigroups, Proc. Nat. Acad. Sci. U.S.A. 42 (1956), 430-432.

UNIVERSITY OF GEORGIA

\section{ON SOME GEOMETRIC INEQUALITIES}

\section{G. D. CHAKERIAN ${ }^{1}$}

1. Let $C$ be a closed curve of class $C^{2}$ in Euclidean $n$-space $E_{n}$. We write the equation of $C$ as $\mathrm{x}=\mathrm{x}(s), 0 \leqq s \leqq L(C)$, where $s$ denotes arc length and $L(C)$ is the length of $C$. Denoting differentiation with respect to $s$ by a dot, we define the total curvature of $C$ as

$$
K(C)=\int_{C}|\ddot{\mathbf{x}}| d s .
$$
then

It is proved in [1] that if $C$ is constrained to lie in a ball of radius $r$,

$$
L(C) \leqq r K(C)
$$

This result is a slight sharpening of an inequality of I. Fáry [2]. The proof given in [1] depends on an integralgeometric lemma for the 2 -dimensional case, together with a reduction of the $n$-dimensional to the 2-dimensional case by developing the curve into a plane. The proof yields no information about curves for which equality occurs in (2).

In $\$ 2$ we give a simple, direct proof of (2) and characterize those curves for which equality holds. We also obtain a sharpening of an inequality of Rešetnjak [3]. A generalization to surfaces is considered in $\S 3$.

Received by the editors July 10, 1963.

1 This research was partially supported by National Science Foundation Grant G-24943. 